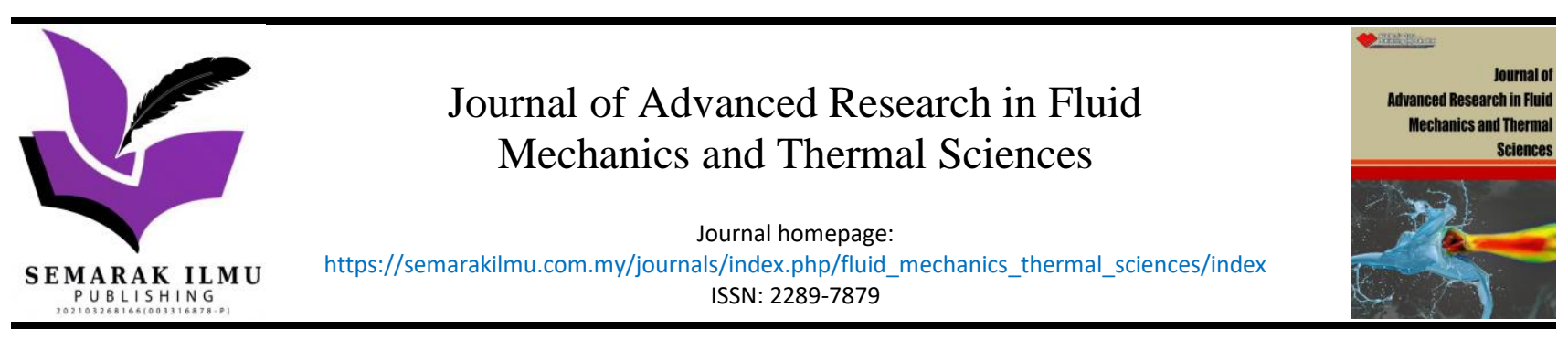

\title{
Study on Aqueous Humour Hydrodynamics of Glaucoma Condition Using 3D Printed Model and Particle Image Velocimetry (PIV)
}

\author{
Riyadhthusollehan Khairulfuaad ${ }^{1}$, Norzelawati Asmuin ${ }^{2,}{ }^{*}$, Ishkrizat Taib $^{2}$ \\ 1 Fakulti Kejuruteraan Mekanikal dan Pembuatan (FKMP), Universiti Tun Hussein Onn Malaysia, Parit Raja, 864000, Malaysia \\ 2 Flow Analysis, Simulation, and Turbulence Research Group (FASTREG), Department of Aeronautic Engineering, Fakulti Kejuruteraan Mekanikal \\ dan Pembuatan (FKMP), Universiti Tun Hussein Onn Malaysia, Parit Raja, 864000, Malaysia
}

\section{ARTICLE INFO}

\section{Article history:}

Received 27 July 2021

Received in revised form 23 September 2021

Accepted 3 October 2021

Available online 16 November 2021

\section{Keywords:}

Particle Image Velocimetry; Glaucoma; Aqueous Humour; 3D model

\section{ABSTRACT}

This study aims to explore the knowledge on fluid flow properties of the aqueous humour (AH), specifically on the anterior segment (AS) of the human eye for a medical condition called Glaucoma. The research objectives are to study on fluid flow characteristics of velocity and pressure of the AH on the AS of the eye using enlarged 3D printed model and computational analysis, and also to analyse the suitability of the 3D generated anterior AS in fluid flow analysis application on particle image velocimetry (PIV). Using polyvinyl alcohol (PVA) as a water-soluble 3D printing filament, a 3D model of the AS of the human eye was generated with SolidWorks 2018 and printed using Creality Ender 3. This printed model serves as the pattern for silicon rubber mould production using vacuum casting process. Analysis of AH flow hydrodynamics are conducted with computational analysis using ANSYS Workbench 19.2. Key findings support that use of PVA material suite the creation of specific shapes and patterns for 3D modelling applications alike, and silicon rubber moulding creates a non-reactive and long-lasting mould for PIV applications. Computational analysis findings support the use of the generated model for PIV applications. Overall, the study successfully supports the use of 3D printed model for PIV application and future work that can be induced include direct experimentation of the mould with PIV.

\section{Introduction}

One of the leading disastrous causes towards blindness is glaucoma and this condition cannot be reversed, only can be stopped. Glaucoma is a medical condition whereby the pressure of the eye is higher than average, a term called high intra ocular pressure (IOP), which causes damage on the optical nerve of a human eye. Causes of this condition includes the poor flow of aqueous humour $(\mathrm{AH})$ in the eye, an eye fluid that is that is generally produced in the ciliary body (CB) and drained off majorly at the trabecular meshwork (TM), and minorly at the uveoscleral pathway as shown in Figure 1.

\footnotetext{
* Corresponding author.

E-mail address: norzela@uthm.edu.my
}

https://doi.org/10.37934/arfmts.89.1.2641 


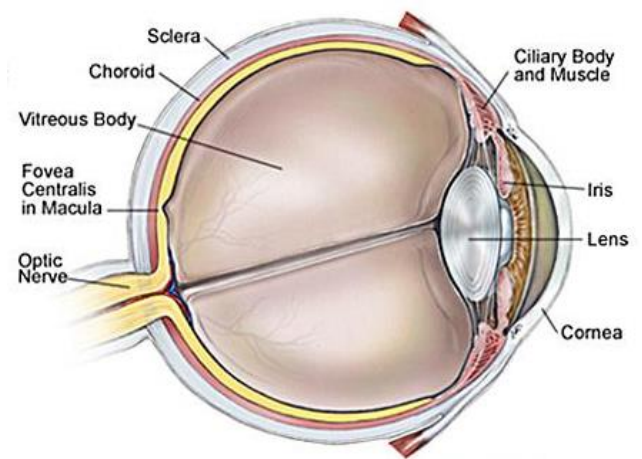

(a)

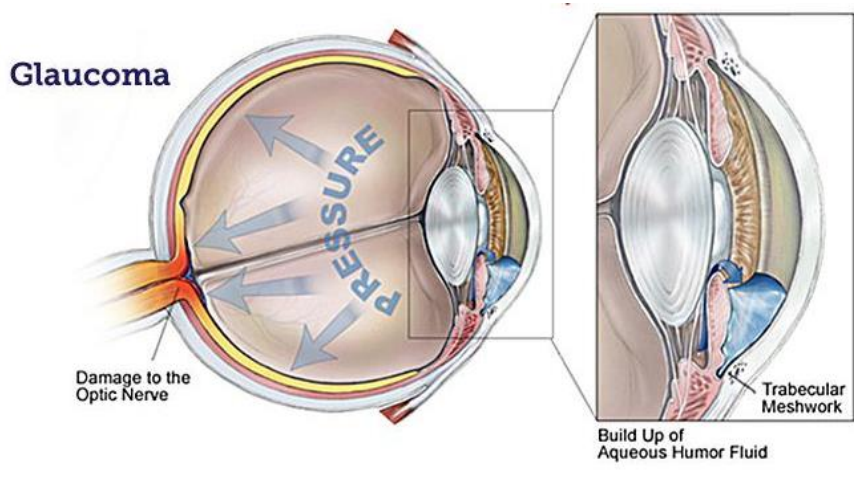

(b)

Fig. 1. Comparison between (a) healthy human eye, and (b) glaucoma eye with damage optic nerves [22]

The efforts of preventing or stopping conditions of glaucoma from deterring includes procedures like inserting a drainage tube called the InnFocus MicroShunt (IMS) procedure, trabeculectomy, laser iridotomy, argon-laser trabeculoplasty and many more [1]. With limited works available on the study in hydrodynamics of the $\mathrm{AH}$ especially on glaucoma conditions, this study aims to set a different perspective and improvement towards analysing the effectiveness of the created 3D model in imaging aqueous humour flow in the anterior segment. The study will consist of a 3D model of the Anterior Segment (AS) of the eye which will be used to simulate the real human eye in glaucoma condition. Computational analysis using ANSYS will then be used to analyse the flow patterns of aqueous humour in the modelled anterior segment.

\subsection{Aqueous Humour and Glaucoma}

The fluids that flow from the posterior chamber to the anterior chamber of the AS is called aqueous humour $(\mathrm{AH})$ and are created from the ciliary epithelium. This fluid is a thin, transparent fluid which is similar to what of a plasma. AH plays role in giving the eye shape through expansion of the cornea, inflating it to look rigid and round. The composition of $\mathrm{AH}$ is $99.9 \%$ water and $0.01 \%$ vitamins, sugar, proteins, and other nutrients [2].

The AH plays a vital role in maintaining overall eye health with functions to maintain IOP by inflating the globes of the eye while also providing nutrients such as amino acids and glucose for the avascular ocular tissues, posterior cornea, trabecular meshwork, lens, and anterior vitreous. $\mathrm{AH}$ is produced by the ciliary body of the anterior chamber, then travels through the iris before being drained at the trabecular meshwork. To maintain proper IOP, the rate of production of $\mathrm{AH}$ must be at the same rate of drainage [3].

The mechanisms involved in the flow of $\mathrm{AH}$ [4]

i. constant replenishment of aqueous fluid from the trabecular meshwork

ii. Phacodonesis (vibration of the human lens when the eye or the head moves)

iii. Rapid Eye Movement (REM) - during the closure of eyelids, the temperature inside the eye is maintained to be uniform everywhere. As a result, the driving force for the natural circulation of $\mathrm{AH}$ (elimination of temp gradient)

iv. Rate of production of $\mathrm{AH}: \pm 2.4 \mathrm{lit} / \mathrm{min}$ (in adults) 


\subsection{3-Dimensional Modelling of The Anterior Segment}

In the medical field, 3D modelling has changed the way researchers imagine the structure and anatomy of the human body. It helps create simulation and software for surgeons to practice virtual surgeries, create direct 3D printed models for studies, and even helped in creating medical tools. 3D printers create successive layers of an appropriate material to create a new version of the subject. With 3D printed models of components and organs such as kidney, there is no longer the need to extract and cover the cost of studying real models obtained from human and even animals [5]. Surgeons also benefit from this technology by creating patient-specific organs to perform practice surgery on before the real procedure. In the study of glaucoma, 3D modelling and printing are used to create a scaled model of the human eye. In this case, previous researchers had already established their scale up human eye model by using this methods and processes [9]. It is generally enlarged as the average size of a real human eye would be complicated to study. Along with the aid of Computational Fluid Dynamics (CFD) and Computer Aided Design (CAD) software, analysis can be conducted towards the 3D printed eye model and its results are concurrently verified from software simulation results. This in return provides comprehensive study on glaucoma and human eye model through imaging analysis and experimental procedure such as particle image velocimetry (PIV).

\subsection{Particle Image Velocimetry (PIV)}

Particle Image Velocimetry (PIV) is one of the current most advanced method to accurately analyses fluid flows [23]. It is a non-intrusive laser optical measurement technique for research and diagnostics into flow, turbulence, microfluidics, spray atomization and combustion processes.

The way PIV works to study and get precise measurements of fluid flow are by taking accurate digital images of seeding particles added to a flowing fluid at one point in time and a second point in time, whilst a laser light illuminates in short pulses towards the measuring sample. These particles when aimed with laser will deflect light, thus being able to be photographed at different times, based on the setting of laser and camera. These images are then analyzed using a computer software to measure the distance and velocity the particles in the flow have travelled [6].

PIV compared to every other available measurement method are at advantage as other methods can only detect velocity at a point, while PIV can take 2-dimensional and even 3-dimensional velocity vectors for the whole area. It is also used as a validation experiment in clarifying Computational Fluid Dynamics (CFD) models as it can measure up to the accuracy that CFD software can achieve.

\section{Materials and Methods}

The focus of this study is to analyse the AH hydrodynamics of the human eye in glaucomatous condition, and to visualize AH flow in human eye. A 3D model of the anterior segment (AS) is to be drawn and be physically 3D printed in order to construct a mould for fluid flow in the AS.

The dimensions of the eye and the primary conditions of analysis are to be determined in order to obtain the most accurate impersonation of the human eye. After obtaining the drawing of the anterior segment, the process then proceeds towards 3D printing model. The model will then be used to create a mould of the AS to allow fluid flow (aqueous humour) through it to simulate the condition similar to when computational simulation is run.

After the model is obtained and simulation results are achieved, the focus is then made towards comparing the manufacturability of the mould in terms of materials and feasibility of such materials 
towards PIV. The simulation results would generally give a visualization on how aqueous humour flow would be when the created model is tested.

\subsection{Determination of Fluid Domain}

The plasma like structure of the $\mathrm{AH}$ has properties similar to that of water therefore physiological $\mathrm{AH}$ can be modeled using saline solution [7]. For the PIV study, normal saline solution will be used as a replacement of $\mathrm{AH}$ for the flow in the created AS mould. This is because of the similarities of the properties of $\mathrm{AH}$ and normal saline solution. Details of the flow parameters use in the study is shown in Table 1.

Since the fluid will be travelling along the silicon mould of the AH model, it has to have a matching refractive index as that of the mould model. If the liquid does not have matching refractive indexes, the direction of illuminating the PIV laser sheet can potentially be deflected as it travels through the silicon [8]. Therefore, a correct composition of glycerine will be added into the saline solution to achieve the same refractive index of the silicon.

\section{Table 1}

Flow parameters of saline solution according to scale

\begin{tabular}{|c|c|c|c|c|c|c|}
\hline Properties & \multicolumn{2}{|c|}{ Original [9] } & \multicolumn{2}{|c|}{ Scaled 5:1 [9] } & \multicolumn{2}{|c|}{ Scaled 10:1 } \\
\hline AH Secretion rate & \multicolumn{2}{|c|}{$1.5-3.0 \mu \mathrm{L} / \mathrm{min}$} & \multicolumn{2}{|c|}{$15 \mu \mathrm{L} / \mathrm{min}$} & \multicolumn{2}{|c|}{$30 \mu \mathrm{L} / \mathrm{min}$} \\
\hline Density, $\rho, \mathrm{kg} / \mathrm{m}^{3}$ & \multicolumn{2}{|l|}{1000} & \multicolumn{2}{|l|}{1000} & \multicolumn{2}{|l|}{1000} \\
\hline IOP/Pressure, & Normal & Glaucoma & Normal & Glaucoma & Normal & Glaucoma \\
\hline $\mathrm{mmHg}$ & 13.5 & 27 & 13.5 & 27 & 13.5 & 27 \\
\hline Viscosity, $\mathrm{kg}^{-1} \mathrm{~s}^{-1}$ & \multicolumn{2}{|l|}{0.001} & \multicolumn{2}{|l|}{0.001} & \multicolumn{2}{|l|}{0.001} \\
\hline Velocity & \multicolumn{2}{|c|}{$1.7684 \times 10^{-4} \mathrm{~ms}^{-1}$} & \multicolumn{2}{|c|}{$1.7684 \times 10^{-5} \mathrm{~ms}^{-1}$} & \multicolumn{2}{|c|}{$1.7684 \times 10^{-5} \mathrm{~ms}^{-1}$} \\
\hline Reynolds No, Re & \multicolumn{2}{|l|}{0.1061} & \multicolumn{2}{|l|}{0.1061} & \multicolumn{2}{|l|}{0.1061} \\
\hline Inlet Diameter & \multicolumn{2}{|l|}{$0.0006 \mathrm{~m}$} & \multicolumn{2}{|l|}{$0.003 \mathrm{~m}$} & \multicolumn{2}{|l|}{$0.006 \mathrm{~m}$} \\
\hline Area, $\mathrm{A}\left(\mathrm{m}^{2}\right)$ & \multicolumn{2}{|c|}{$2.8274 \times 10^{-7}$} & \multicolumn{2}{|c|}{$2.8274 \times 10^{-6}$} & \multicolumn{2}{|c|}{$2.8274 \times 10^{-5}$} \\
\hline
\end{tabular}

Using temperature dependent calculation method to determining the refractive index of the required solution based on Eq. (1)

$n_{\text {target }}=p\left(a_{1}+b_{1} T\right)+(1-p)\left(a_{2}+b_{2} T\right)$

where

$n_{\text {target }}:$ Refractive index of mixture

$p$ : proportion of glycerine

$\mathrm{T}:$ Temperature (Kelvin)

$a_{1}, b_{1}, a_{2} \& b_{2}:$ Empirical constants specific to glycerine and water

By setting T at 298K, $a_{1}, b_{1}, a_{2} \& b_{2}$ as $1.3774,-0.0002,1.5463$, and -0.0002 ; it was noted that the refractive index of the AS Silicon Rubber mould was 1.55 [8]. By setting the $n_{\text {target }}$ as 1.55 , the value of ' $p$ ' or the percentage of Glycerine required is 0.1940 . Thus, to match the refractive index of the silicon rubber mould to water at room temperature, a mixture of $19.4 \%$ glycerine to $80.6 \%$ water is to be used the conduct a PIV study. 


\subsection{D Modelling of the Anterior Segment}

The modelling of the AS of the human eye was constructed using computer aided design software SolidWorks 2018. The parts that are drawn for the AS includes part of the posterior cornea, lens, iris, and pupil opening. The focus of the 3D modelling is to get chamber where AH travels in the eye to get a 3D print of the model to create mould of the AS. Modelling the eye follows the following parameters in Table 2.

Table 2

Parameters of the AS Model

\begin{tabular}{|c|c|c|c|}
\hline \multirow[t]{2}{*}{ Quantity } & \multicolumn{3}{|l|}{ Dimension } \\
\hline & PIV Model & Reference Model [9] & Original Model [9] \\
\hline Scaling Factor & $10: 1$ & $5: 1$ & $1: 1$ \\
\hline $\begin{array}{l}\text { Diameter of the anterior chamber, Dc, } \\
\mathrm{mm}\end{array}$ & 130 & 65 & 13 \\
\hline Maximum height of chamber, hc, mm & 26.3 & 13.15 & 2.63 \\
\hline $\begin{array}{l}\text { Maximum radius of curvature of the } \\
\text { posterior cornea, } \mathrm{Rc}, \mathrm{mm}\end{array}$ & 68 & 30.0 & 6.8 \\
\hline $\begin{array}{l}\text { Radius of curvature of the natural lens, } \\
R_{L}, \mathrm{~mm}\end{array}$ & 100 & 50 & 10 \\
\hline Height of the iris-lens channel, $\mathrm{mm}$ & 1.0 & 0.5 & 0.1 \\
\hline Angle between cornea and iris, $\underline{0}$ & 30 & 300 & 30 \\
\hline
\end{tabular}

\subsection{Production of Anterior Segment Model and Silicon Rubber Mould}

Anterior segment of the glaucomatous eye was modelled in SolidWorks2018 at 10-times scaledup. The 3D model was saved as .st/ format (format native to the stereolithography CAD software created by 3D Systems) and then transferred into commercial 3D printer software known as Ultimaker Cura version 4.1.0. The 3D model was then saved in a .gcode format (format mainly used in Computer-Aided Manufacturing to control automated manufacturing tools) before being transferred into 3D printer's Arduino. A standard operation procedure (SOP) of the 3D printer is carried out before printing. These include the levelling process, filament extruder test, and temperature test. The material polyvinyl alcohol (PVA) filament is used for the print.

PVA filament material has the ability to dissolve in water, thus this factor is the reason that material is used to create the AS inner pattern, so it would dissolve in water during the mould fabrication process.

3D printer model was a CREALITY Ender 3. The 3D printer was set to print when temperature of extruder reaches $200^{\circ} \mathrm{C}$ with standard bed's temperature for the material. The same method is used for the AS outer pattern of the AS model but printed using a different material called polylactic acid, PLA. After the 3D print model is completed, finishing process of the model is carried out to ensure that the surface of the model is smooth and does not promote any rough or dented surface. Figure 2 shows the process flow of 3D printing the AS model from CAD file to physical model.

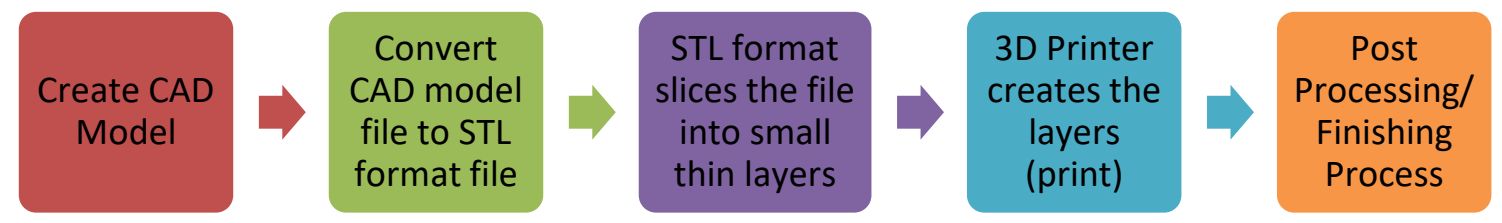

Fig. 2. Process flow of AS pattern creation 
Silicone rubber is used for the mould making process. The AS outer pattern is placed inside a container with holder to fix its place. Once the structure is set up in place, the mixture of transparent silicone rubber is slowly poured into the container to avoid air bubble formation in any possible causes by turbulence flow of the silicone. This is done by using vacuum casting process. Air bubbles must be ensured to not form in the mould as it would affect and or damage the images taken during PIV study. The air vacuum compressor is used to remove the air bubbles inside the silicone mixture. After all air bubble is removed, let the mould solidify at its own rate. After the mould is fully solidified, the AS pattern is removed from the silicone rubber. This is done by fully submerging the AS transparent silicone in warm water to let the PVA printed pattern dissolve. Finishing process for the transparent silicone AS model is carried out before the model is air dried and ready for analysis and PIV analysis application.

\subsection{Computational Analysis using ANSYS Workbench 19.2}

The process of conducting computational fluid analysis follow basic steps as available in ANSYS Fluent of the ANSYS Workbench 19.2. For this study the geometry that will be used to run the analysis will be the drawn 3D model of the anterior segment. From a SolidWorks .SLDPRT file, conversion is done to make it a STEP (Standard for the Exchange of Product Data) file which is readable by ANSYS.

After the 3D model drawing of the anterior segment is inserted into the software, the simulation 'Setup' is then adjusted. The dimension settings set at ' $3 \mathrm{D}$ ', while other settings will be remained as default. In the 'Fluidzone', the property of the fluid will be defined, whereby the values of density, specific heat, thermal conductivity and also viscosity of aqueous humour is input into the system. Boundary conditions are set at the inlet, the interior fluidzone, the outlet, wall, wall anterior iris and also wall posterior iris. After defining standard inputs for the simulation, select 'Run Calculation' with time stepping method set at 'Fixed' at time step 0.5 seconds and 20 number of time steps.

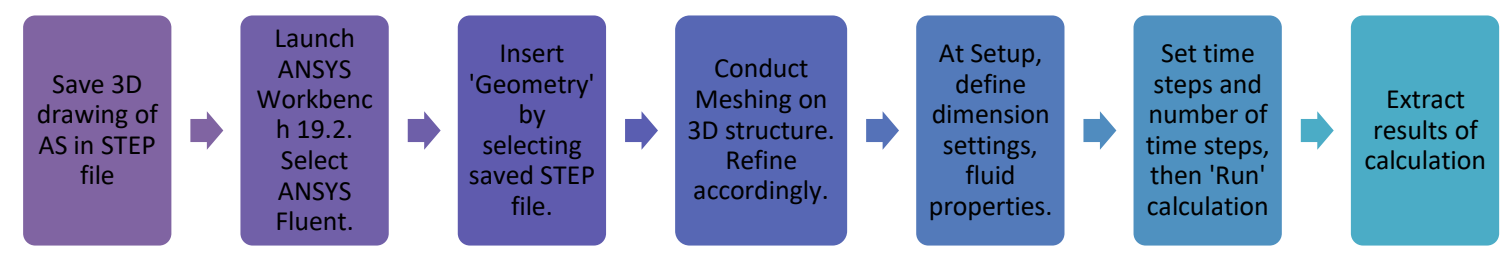

Fig. 3. Flow process of setting up the computational analysis

\section{Results and Discussion}

\subsection{3-Dimensional Model of the Anterior Segment}

By modelling the anterior segment on SolidWorks 2018, a complete 3D model is obtained using the dimensions from Table 2. Figure 4 below shows the modeled AS in SolidWorks 2018. 


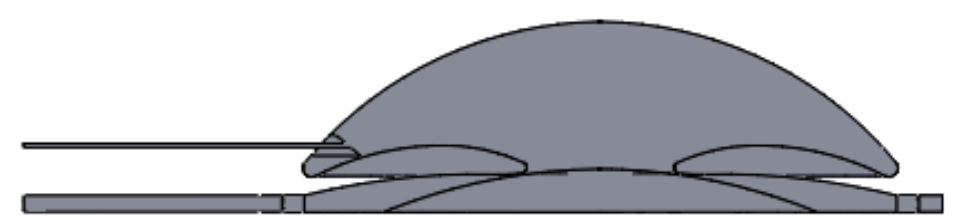

(a)

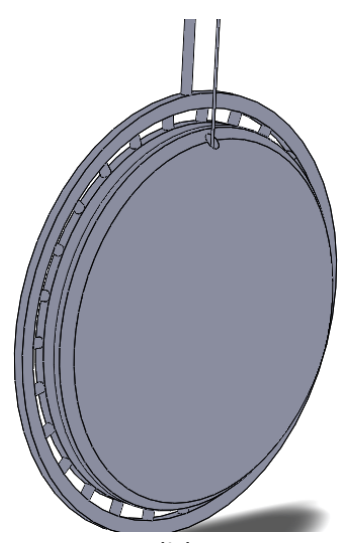

(b)

Fig. 4. The modelled anterior segment, AS of (a) Cross Sectional view of anterior segment, and (b) Full drawing of anterior segment

Using the Creality Ender 3 printer and PVA filament, the model of the AS was printed at an enlarged 10:1 Scale. The outcome from the printing is shown in Figure 5(a), Figure 5(b), Figure 6 and Figure 7 below.

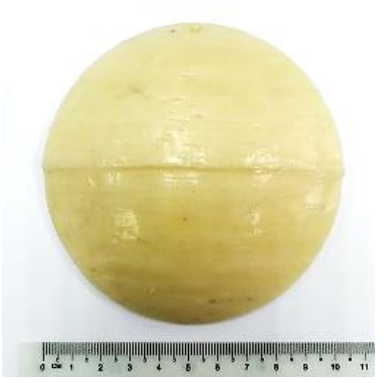

(a)

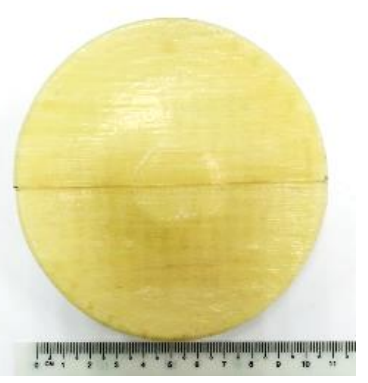

(b)

Fig. 5. The anterior chamber PVA printed using 3D printer machine, (a) front view, and (b) back view

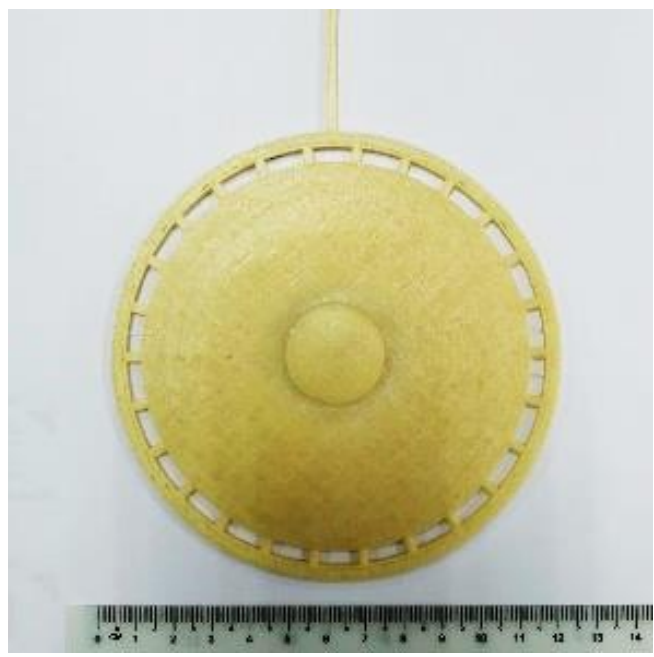

Fig. 6. Printed Posterior Chamber of Anterior Segment 


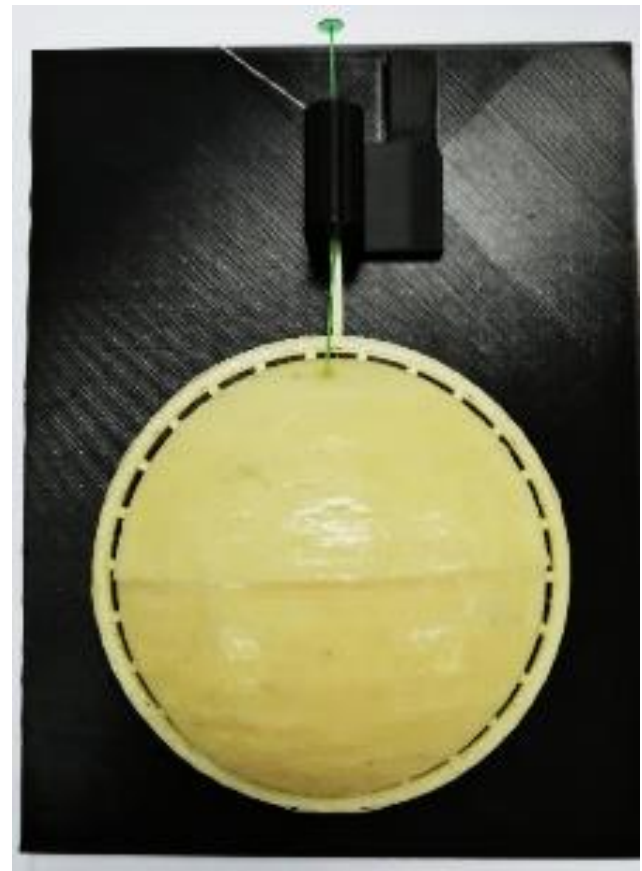

Fig. 7. Attached model for Silicon Rubber mould making

Based on the images of the printed 3D model using PVA, it is observed that the model was printed on a total of 3 separate parts. The support structure of the printed model is of standard settings and at $20 \%$ support density. These parts are then glued together to create the complete model of the anterior segment.

The processes from mixing to pouring and vacuum casting of silicon rubber into the encased PVA model was done in compliance to the working time of the Shin Etsu KE-1603 RTV Silicon Rubber. Table 3 shows the properties of the silicon rubber.

Table 3

Product Details of Shin Etsu 2-Component RTV Silicon Rubber Model KE-1603 (10)

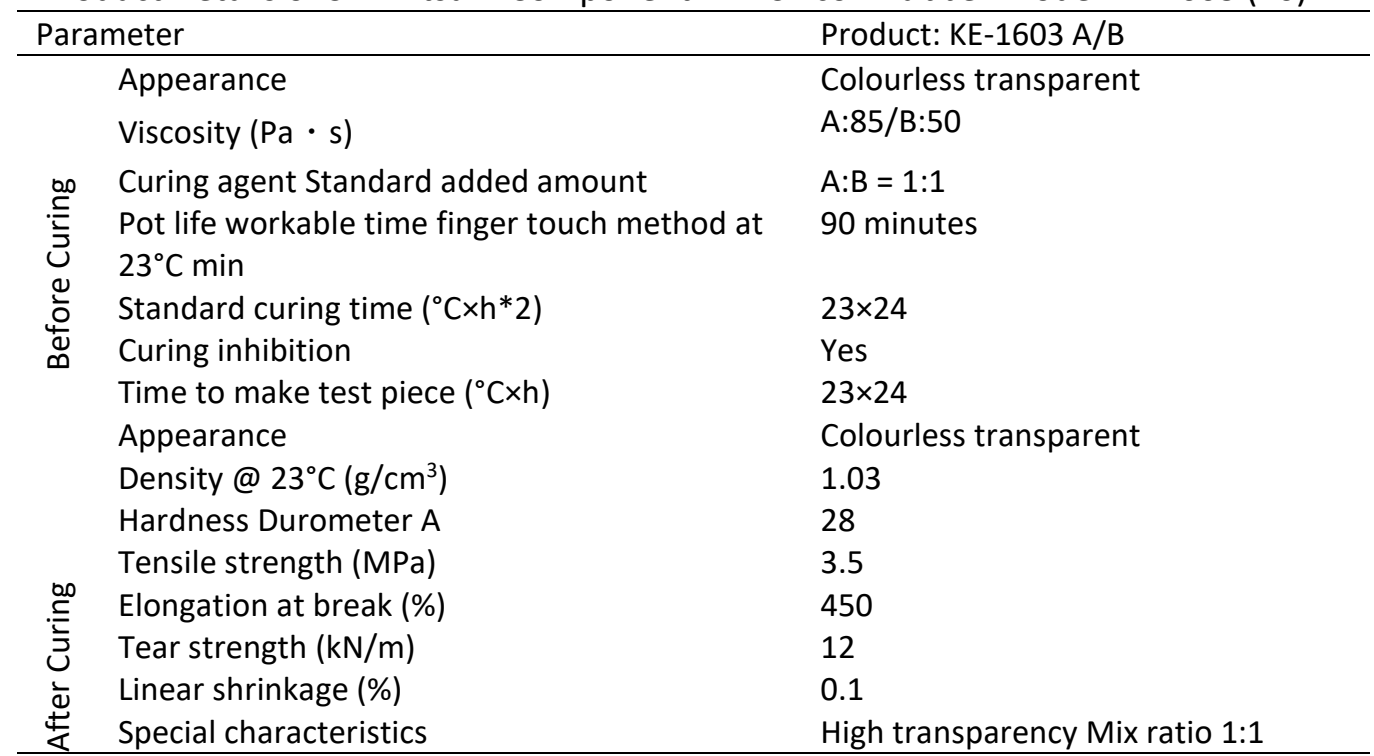


The completed AS mould was then left in a cool dry room at standard room temperature to completely cure and solidify. This high-strength addition cure silicon with a product of high transparency also features good dimensional stability, whereby in the process of curing, the shrinkage that occurs are minimal, and the non-exothermic process would deter any additional effects that may occur towards the original model [11]. As observed on Figure 8(a), it is noted that the 3D printed PVA AS model managed to stay in place throughout the process.

However, the support for the solid component at the anterior chamber has collapsed as shown in Figure 8(b). This is possibly due to the reaction towards high changes in pressure in the vacuum casting machine, followed by the high viscosity of the working process of silicon rubber acting around the model. As the support structures printed in the anterior chamber were standard supports only at $20 \%$ density, clearly the results after vacuum casting shows it was insufficient to withstand the process.

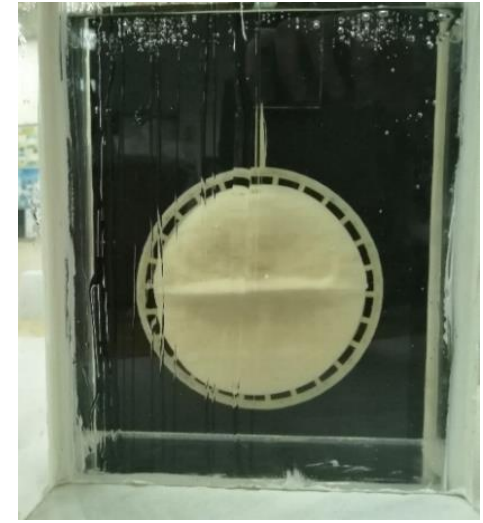

(a)

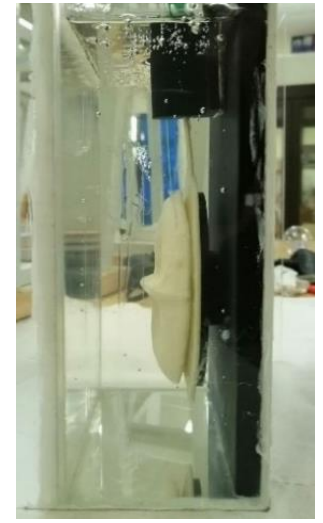

(b)

Fig. 8. The Silicon mould after vacuum process in vacuum chamber (a) front view (b) right view

The process of removal of the PVA Model of the AS after creation of the silicon mould was done by consistently flowing warm water at approximately $35^{\circ} \mathrm{C}$ to increase dissolving rate [12]. A water pump of 15-Watt power, branded KINTONS Eco Green Series Model KT102 with a maximum flow rate of $1200 \mathrm{~L} /$ Hour was used to drive water towards the inlets of the silicon model.

The following Figure 9(a), and Figure 9(b) shows the silicon model before the PVA model was dissolved, and after the PVA model was fully dissolved. 


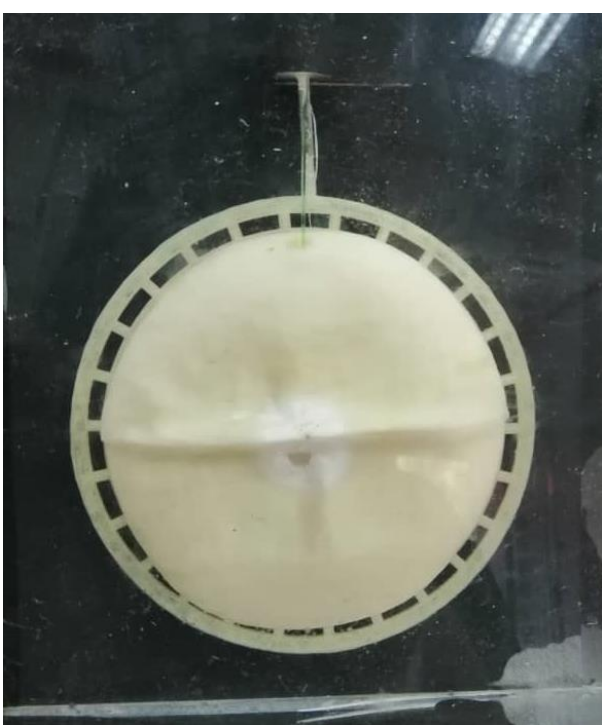

(a)

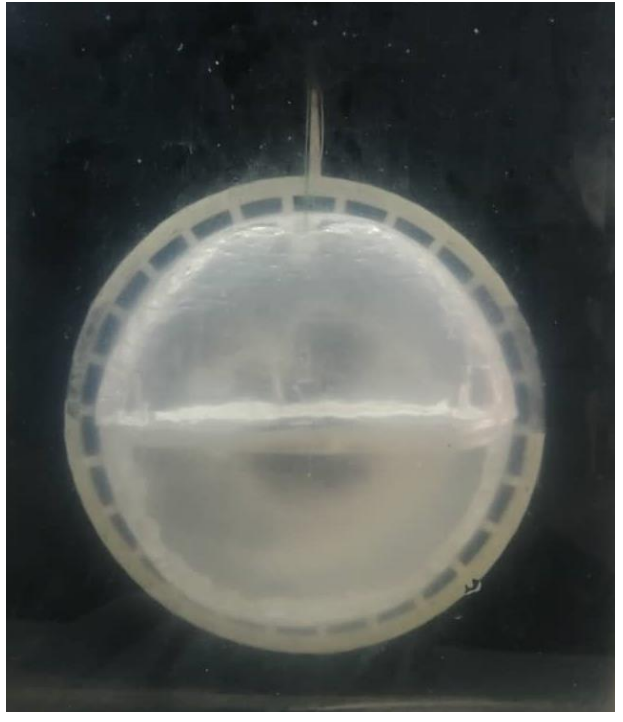

(b)

Fig. 9. AS Mould (a) before, and (b) after PVA removal

\subsection{Variation of Pressure and Velocity in Anterior Segment Model}

By computational analysis using ANSYS, the surfaces of the mesh were first defined to ensure better encapsulation of data, and surfaces of the anterior and posterior iris were defined separately. The Figure 10 below shows the complete meshed fluid zone of the AS for computational analysis and the standard conditions set for the computational analysis are stated in Table 4.

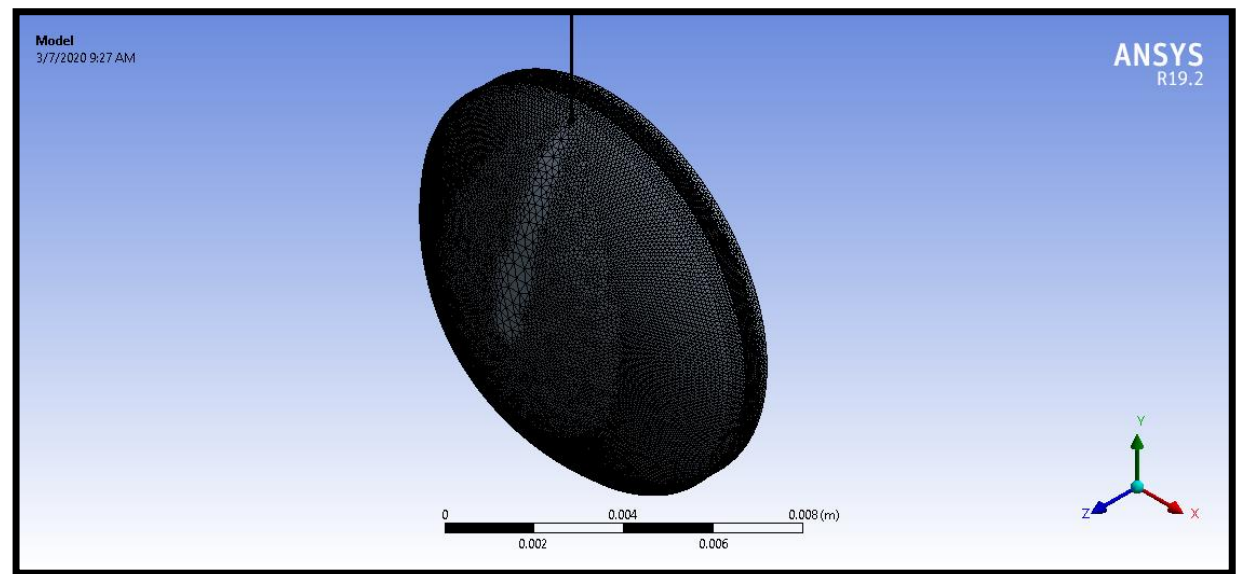

Fig. 10. Meshing of the AS Model

Table 4

Parameters Set for Computational Analysis on ANSYS Workbench 19.2

\begin{tabular}{ll}
\hline Parameters & Value \\
\hline Mass flow rate $(\mathrm{kg} / \mathrm{s})$ & $5 \times 10^{-8}$ \\
Inlet Pressure $(\mathrm{Pa})$ & 3599 \\
Outlet Pressure $(\mathrm{Pa})$ & 1733 \\
Fluid Viscosity $(\mathrm{kg} / \mathrm{ms})$ & 0.001 \\
Fluid Density $\left(\mathrm{kg} / \mathrm{m}^{3}\right)$ & 1000 \\
\hline
\end{tabular}




\subsubsection{Distribution of pressure along the AS}

In the present study of the simulation of the AH flow in the AS it was noted that the distribution of pressure along the model is average throughout the model, as shown in the pressure contour in Figure 11. In accordance to the Y-axis, the flow pressure is consistent at $4719 \mathrm{~Pa}$ throughout the anterior and posterior chambers. This shows that the experimental model when simulated has a slightly higher AH flow pressure, than the referred $27 \mathrm{mmHg}(3599.7 \mathrm{~Pa})$ of the referenced model. This can be related to the absence of outflow channels in the drawn model, as only there one outlet is set for the experimental model to ease the process of acquiring data from PIV analysis. The distribution of pressure is then slowly decreasing towards the outlet.

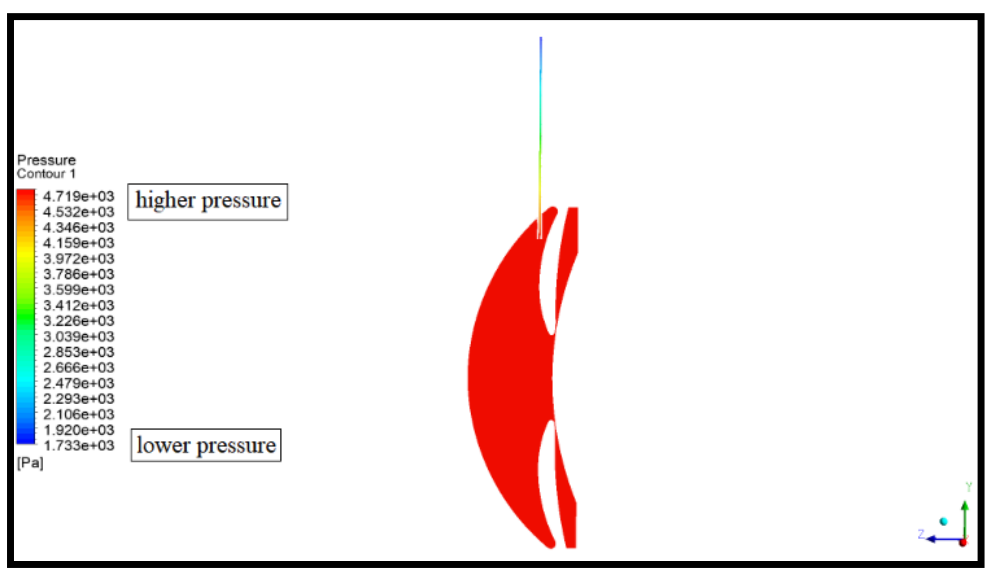

Fig. 11. Distribution of Pressure Along Y Axis of The AS Model

\subsubsection{Velocity vector of $A H$ flow in AS}

In accordance to flow velocity vector, Figure 12 below shows the distribution of velocity vector along Y-axis of the AS model. The flow velocity is relatively low, as the flow rate of AH in a human eye is about $3.0 \mu \mathrm{L} / \mathrm{min}$. The flow of $\mathrm{AH}$ enters the anterior segment through the iris-lens gap, and as it travels to the centre it then flows out to the anterior chamber from the pupil, before spreading out to the whole anterior chamber, and reached the walls of the cornea before leaving at the trabecular meshwork, which in this simulation model, is at the outlet of the model at the top.

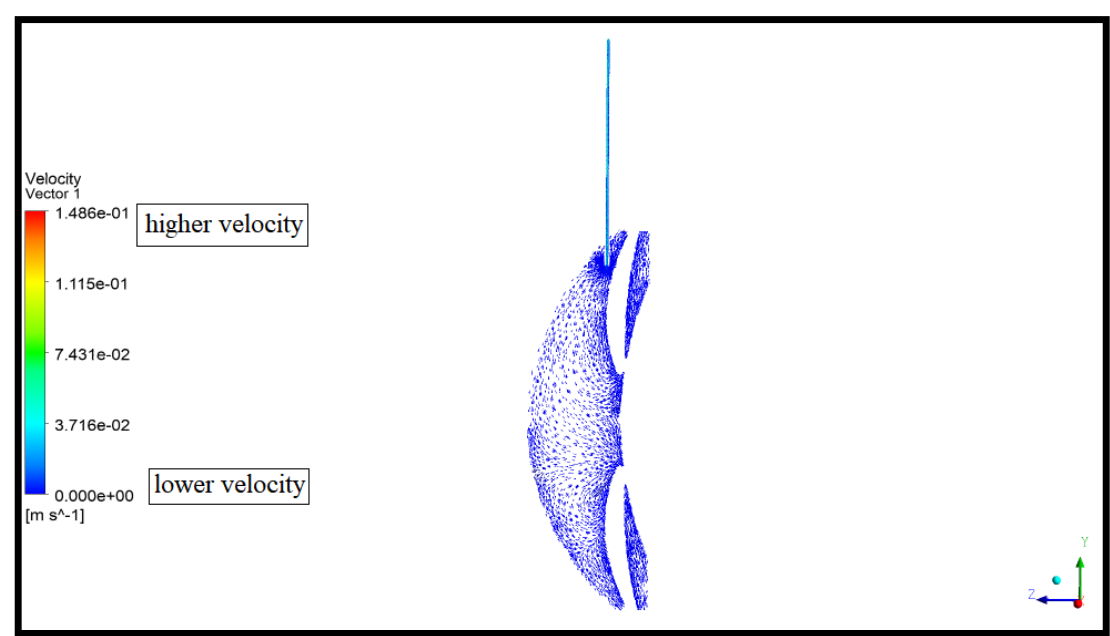

Fig. 12. Distribution of Velocity Vector Along Y Axis of The AS Model 
From Figure 13 below we may observe the flow separation at the anterior chamber of the eye, which occurs at a lower than centre line of the AS model. The separation happening at the lower region of the AS model suggests more AH flows upwards towards the outlet channel after entering the anterior chamber.

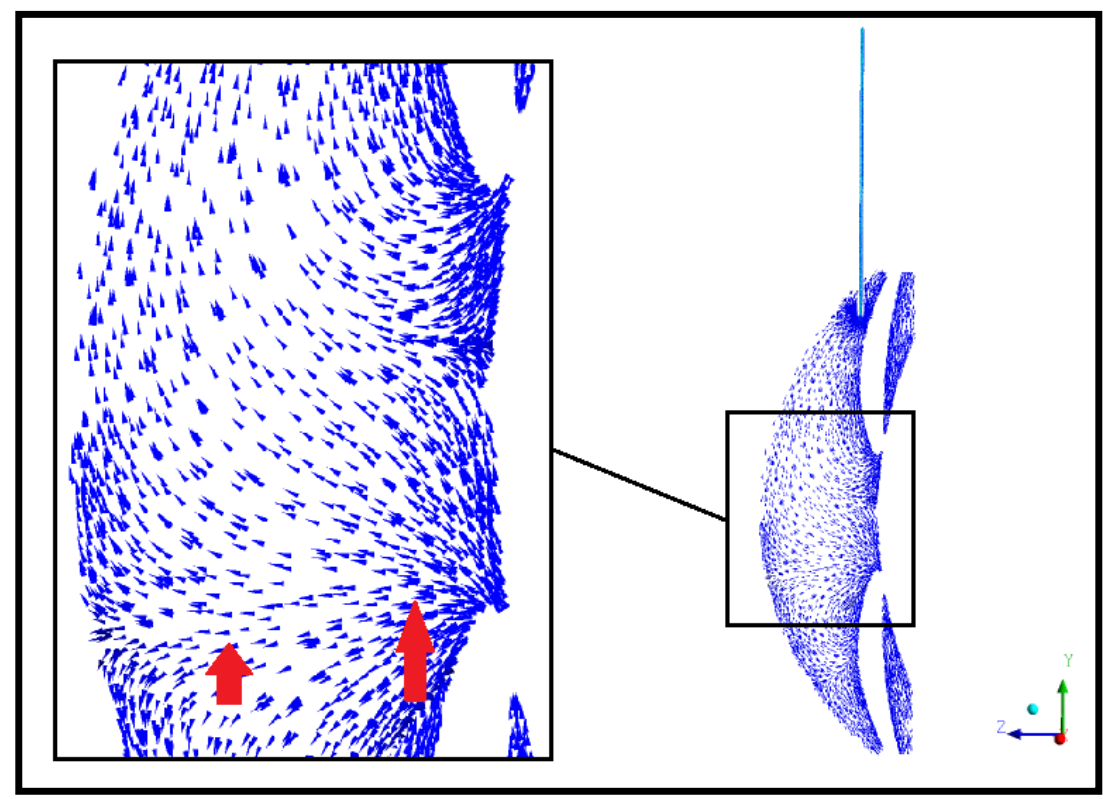

Fig. 13. Flow Separation of AH in the AS Model

The happenings of backflow in the AS model also occurs at the lower segment as there are no flow exit chambers at the lower region of the model. Figure 14 shows the happenings of backflow in the AS model.

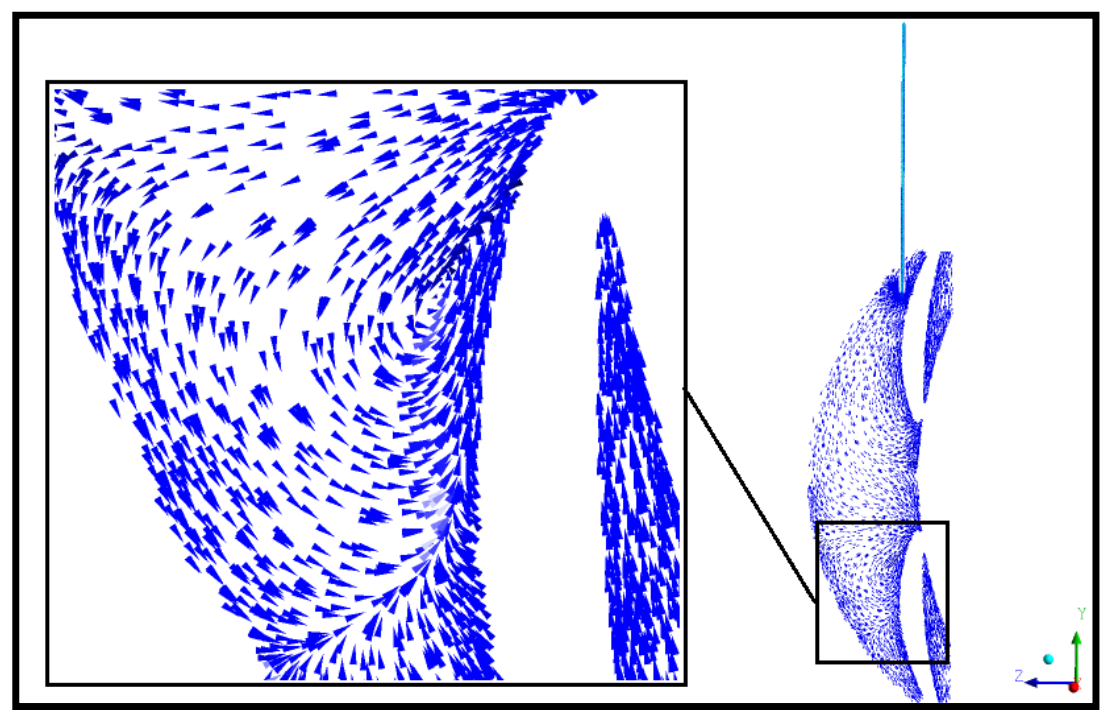

Fig. 14. Backflow in AS Model

\subsection{Comparison of Materials for Anterior Segment 3D Printing and Mould Making}

Material PVA was selected based on its extended use as a 3D print material that is easily removed, just by using water [13]. This may be true, but the processes of removing the 3D printed AS model made of PVA was a time-consuming process in the creation of this AS mould due to the inability to 
insert physical force to speed up the process of removal. To add on, there were no other chemical solution that can help speed up the process. The material is also costly due to its manufacturability, and its solubility properties depends on the manufacturer's specifications [14]. Thus, not all PVA filaments react at the same speed when exposed to water.

Other materials that can be considered for printing the AS model, such as HydroFill, Infinity, and SUP 706 which are also water-soluble materials. Experimenters can also opt to use newly developed materials like AquaSys which reacts faster to water than normal PVA filaments [15]. Soluble materials ease the user by providing hands free methods of removal, or those that only require minimal scrubbing and abrasive brushing which in turn saves time. Similarly, even if different soluble materials are used for printing, it still takes some time for the material to dissolve completely, and this is attributed also by the density of the 3D print.

Nevertheless, despite the abundance of different 3D print materials that are better than PVA, there are hardly any references that can be taken into consideration when creating a part of the anatomy of the human body. For example, the creation of a cerebral aneurysm by Ho et al., [16], or patient-specific vascular anatomies by Ruedinger et al., [17], and many more biomedical applications of transparent mould making uses the material PVA to create their 3D patterns.

The processes of vacuum casting with silicone rubber moulding were chosen as it has the ability to capture the detail and intricateness of the model, capable of reproducing extremely complex geometries with very fine details [18]. As the printed PVA model was encased within hard, solid, transparent silicon rubber, the process of physically removing the printed PVA took much of mould preparation time as physical force such as scrubbing was impossible to be imposed due to small channels of inlet and outlet of the AS model.

The material silicon rubber was selected based on various past works as silicon rubber mould has a clear and transparent look, and it has an acceptable refractive index, which can be matched by appropriate solution of water to glycerine ratio [8]. This enables the possibility to conduct PIV study on the model as requirements are achieved. The processes of vacuum casting the silicon rubber mould from the AS pattern also creates a nice finish, as air bubbles are not present on the mould.

Comparatively, another material named plexiglass are also suitable for mould making for PIV applications. Plexiglass is a material made out from the methyl methacrylate polymer, which is an ester or methacrylic acid. It has a clear appearance, and although it is plastic, it is a great replacement of glass as it is shatter proof, strong and flexible [19]. By modelling using Computer Numerical Control (CNC) machining and conducting refractive index matching for PIV flow solution, plexiglass is also proven as great materials for mould making for PIV applications. In the essence of conducting PIV studies, Table 5 below shows the comparison between two of the common materials used to create moulds.

Table 5

Comparison in use of Silicon Rubber and Plexiglass for Manufacture of PIV Experiment Mould

\begin{tabular}{lll}
\hline Silicon Rubber & Parameter & Plexiglass [20] \\
\hline $\begin{array}{l}\text { Lost-pattern Vacuum Casting } \\
\text { (dissolvable pattern) }\end{array}$ & Manufacturing & $\begin{array}{l}\text { Computer Numerical Control } \\
\text { (CNC) Machining }\end{array}$ \\
Colourless transparent & Appearance & Colourless and clear \\
$1030 \mathrm{~kg} / \mathrm{m}^{3}$ & Density & $1190 \mathrm{~kg} / \mathrm{m}^{3}$ \\
$3.5 \mathrm{MPa}$ & Tensile Strength & $80 \mathrm{MPa}$ \\
1.55 & Refractive Index & 1.491 \\
$\mathrm{NO}$ & Susceptible to scratches / shatter & Yes \\
High (RM \pm 2500$)$ & Manufacturing cost & High (RM \pm 5000$)$ \\
Created Model & & \\
\hline
\end{tabular}


From the generated table above, it is consenting that both these materials come in handy for the experimentation of PIV, due to their colourless transparent appearance that enables light to pass through completely. In addition, both refractive indexes of the materials are relatively low, suggesting it is generally easier for practitioners to match the refractive index of the working fluid to those of the model which eases the process of conditioning the mould for PIV before obtaining data.

Although both materials serve similar benefits, their chemical properties result in different physical states. Silicon rubber moulds are firm, yet to the touch it gives a very minimal feel of compression as those where one can get from touching a rubber shoe sole. Plexiglass on the other hand has solid, rigid plastic feel. Due to this, plexiglass is more prone towards scratches when placed on rough surfaces, and they may also break or shatter when excessive force is imposed. Besides, any misalignment while CNC machining the plexiglass may lead to micro shatters which will then escalate towards a large crack as heat from PIV laser builds up when the mould is continuously used [21]. Unlike silicon rubber, as it is more adaptable towards external pressures, moulds made of such materials tend to last longer and are more economical.

\section{Conclusion}

This study was conducted with the idea of exploring the aqueous humour flow characteristics of velocity and pressure for primary angle closure glaucoma cases, using a created 3-dimensional mould of the anterior segment and also running computational analysis with ANSYS Workbench 19.2. From obtained results, it was noted that the support density of the 3D printed anterior segment at $20 \%$ density was unable to sustain the changes in pressure during vacuum casting process as the model collapsed internally after being moulded. Thus, the support density of the 3D print should be increased for future manufactures. Based on ANSYS simulation results with standard primary angle closure glaucoma conditions set at inlet flow of $5 \times 10-8 \mathrm{~kg} / \mathrm{s}$ and inlet pressure of $3599 \mathrm{~Pa}$, it was noted that there is slight rise in pressure of flow for the simulated model, whereby the obtained pressure was $4719 \mathrm{~Pa}$, which is higher than expected. The availability of only one outlet from the generated anterior segment model contributed to the rise in pressure, while also the results in velocity vector of the computational analysis shows signs of backflow the lower region of the model. When compared in manufacturing and material, the material used for 3D printing the anterior segment model, which was polyvinyl alcohol (PVA) shows significant specialties as not only it can be used with standard 3D printers, but it is also water soluble which makes it a great material for various mould making applications for patterns with complex designs. In terms of creating a mould for PIV applications, the created silicon rubber mould not only presents high transparency, yet its ability for easy refractive index matching and its non-reactivity towards physical disturbances like heat and force makes it a great material for creating long lasting moulds which can be used repeatedly.

Overall, the study conducted hopes to bring an in-depth knowledge on the flow properties aqueous humour on the condition of glaucoma, with the use of proper technology of 3-dimensional modelling, rapid prototyping and also make good use analysis methods like computational analysis using ANSYS and also particle image velocimetry (PIV).

\section{Acknowledgement}

This research was made possible by support from the Ministry of Higher Education (MOHE) Malaysia and Universiti Tun Hussein Onn Malaysia (UTHM) internal grant of Postgraduate Research Grant (GPPS) vot H036 and Multidisciplinary Grant (MDR) vot H500. The authors would also like to thank the Faculty of Mechanical and Manufacturing Engineering, the Flow Analysis, Simulation, and 
Turbulence Research Group (FASTREG) research group of Universiti Tun Hussein Onn Malaysia for its support.

\section{References}

[1] Glaucoma. "The Path To A Cure for Glaucoma." Glaucoma Research Foundation. Accessed October 9, 2019. https://www.glaucoma.org/research/the-path-to-a-cure-for-glaucoma.php.

[2] Vision Eye Institute. "The aqueous humour." Vision Eye Institute. August, 262017. https://visioneyeinstitute.com.au/eyematters/aqueous-humour/.

[3] FLEI. "Aqueous Humor and its Role in the Eye." Fort Lauderdale Eye Institute. Accessed October 31, 2019. https://flei.com/aqueous-humor-and-its-role-in-the-eye/.

[4] Ooi, Ean-Hin, and Eddie Yin-Kwee Ng. "Simulation of aqueous humor hydrodynamics in human eye heat transfer." Computers in Biology and Medicine 38, no. 2 (2008): 252-262. https://doi.org/10.1016/i.compbiomed.2007.10.007

[5] Kusaka, M., M. Sugimoto, N. Fukami, H. Sasaki, M. Takenaka, T. Anraku, T. Ito, T. Kenmochi, R. Shiroki, and K. Hoshinaga. "Initial experience with a tailor-made simulation and navigation program using a 3-D printer model of kidney transplantation surgery." In Transplantation Proceedings, vol. 47, no. 3, pp. 596-599. Elsevier, 2015. https://doi.org/10.1016/i.transproceed.2014.12.045

[6] Atkins, M. D. "Velocity field measurement using particle image velocimetry (PIV)." In Application of Thermo-Fluidic Measurement Techniques, pp. 125-166. Butterworth-Heinemann, 2016. https://doi.org/10.1016/B978-0-12809731-1.00005-8

[7] Wang, Wenjia, Xiuqing Qian, Hongfang Song, Mindi Zhang, and Zhicheng Liu. "Fluid and structure coupling analysis of the interaction between aqueous humor and iris." Biomedical Engineering Online 15, no. 2 (2016): 569-586. https://doi.org/10.1186/s12938-016-0261-3

[8] Yip, Ronnie, Rosaire Mongrain, Adrian Ranga, Jean Brunette, and Raymond Cartier. "Development of anatomically correct mock-ups of the aorta for PIV investigations." Proceedings of the Canadian Engineering Education Association (CEEA) (2004).

[9] Wang, Wenjia, Xiuqing Qian, Qi Li, Gong Zhang, Huangxuan Zhao, Li Tan, Yu Yang, Hongfang Song, and Zhicheng Liu. "Experimental study of aqueous humor flow in a transparent anterior segment phantom by using PIV technique." Molecular \& Cellular Biomechanics 16, no. 1 (2019): 59-74. https://doi.org/10.32604/mcb.2019.06393

[10] Shin Etsu. "RTV Silicone Rubber for Mouldmaking." Shin Etsu Silicone Global. 2002. https://www.shinetsu.co.jp/en/products/functional-materials/rtv-silicone-rubbers-for-moldmaking/.

[11] Shin Etsu. "Two-component RTV rubbers for moldmaking." Shin Etsu Silicone Global. 2020. https://www.shinetsusilicone-global.com/products/type/two rtv mold/index.shtml.

[12] Ultimaker. "How to dissolve Ultimaker PVA." Ultimaker Support. June 29, 2021. https://support.ultimaker.com/hc/en-us/articles/360011959100-How-to-dissolve-Ultimaker-PVA.

[13] Simplify3D. "PVA." Simplify3d ${ }^{\oplus}$. 2020. https://www.simplify3d.com/support/materials-guide/pva.

[14] Carolo, Lucas. "PVA Filament - Best Brands in 2020." All3DP. July 18, 2020. https://all3dp.com/2/pva-filamentexplained-and-compared/.

[15] Team Infinite Materials. "Advancements in Water-Soluble Materials for 3D Printing Support Filament." 3D Printing Materials. November 12, 2019. https://3dprint.com/259055/advancements-in-water-soluble-materials-for-3dprinting-support-filament/.

[16] Ho, W. H., I. J. Tshimanga, M. N. Ngoepe, M. C. Jermy, and P. H. Geoghegan. "Evaluation of a desktop 3D printed rigid refractive-indexed-matched flow phantom for PIV measurements on cerebral aneurysms." Cardiovascular Engineering and Technology 11, no. 1 (2020): 14-23. https://doi.org/10.1007/s13239-019-00444-z

[17] Ruedinger, Katrina L., Rafael Medero, and Alejandro Roldán-Alzate. "Fabrication of low-cost patient-specific vascular models for particle image velocimetry." Cardiovascular Engineering and Technology 10, no. 3 (2019): 500507. https://doi.org/10.1007/s13239-019-00417-2

[18] Salleh, Zulzamri, and M. Yuzri M. Yusop. "Design and fabrication of silicon rubber mould (SRM)." International Journal of Mechanical Engineering 2, no. 3 (2013): 63-68.

[19] Joram, Christian. Transmission curves of plexiglass (PMMA) and optical grease. No. PH-EP-Tech-Note-2009-003. 2009.

[20] SONGHAN. "Evonik Corporation Plexiglas ${ }^{\circledR}$ GS OF00 (222) Cast Acrylic." Look Polymers. 2020. https://mychem.ir/uploads/tds/16457.pdf.

[21] Giurgea, Corina, Florin Bode, Lucian Nascutiu, and Cristian Dudescu. "Considerations regarding the optically transparent rigid model for PIV investigations. A case study. Part 2: Notes on the failure of the model." Energy Procedia 85 (2016): 235-243. https://doi.org/10.1016/i.egypro.2015.12.233 
[22] Bright Focus Foundation. "How the Build Up of Aqueous Humor Can Damage the Optic Nerve." BrightFocus ${ }^{\circledR}$. July 21, 2019. https://www.brightfocus.org/glaucoma/infographic/how-build-aqueous-humor-can-damage-opticnerve.

[23] Othman, Safiah, Abas A. Wahab, and Vijay R. Raghavan. "Validation by PIV of the numerical study of flow in the plenum chamber of a swirling fluidized bed." CFD Letters 2, no. 2 (2010): 85-96. 